\title{
Film Formation and Anticorrosive Behavior of Zn-ZSM-5 Nano-Sized Zeolite Composite Coatings
}

\author{
Hamid Reza Safaei ${ }^{1, *}$, Mohammad Reza Safaei ${ }^{2}$ and Vahid Rahmanian ${ }^{3}$ \\ ${ }^{I}$ Department of Applied Chemistry, Faculty of Science, Shiraz Branch, Islamic Azad University, PO Box 71993-5, \\ Shiraz, Iran \\ ${ }^{2}$ Department of Practical Science, Estahban Branch, Islamic Azad University, Estahban, Iran \\ ${ }^{3}$ Department of Industrial Polymer Engineering, Shiraz Branch, Islamic Azad University, Shiraz, Iran
}

\begin{abstract}
The zinc- Zeolite Socony Mobil-Five nano-particles (Zn-ZSM-5 NPs) composite was prepared and applied successfully as a new coating material to improve the corrosion resistance of steel. The corrosion type and quality of both pure zinc and our new coating material, Zn-ZSM-5 NPs, were studied using mass loss, impedance and electro-chemical polarization techniques in a sodium chloride solution. Obtained results affirm that Zn-ZSM-5 NPs are non-permeable and their corrosion resistance is higher than the pure zinc. The surface morphology of our newly synthesized coating material, Zn-ZSM-5 NPs, was investigated using SEM and X-ray diffraction and the smaller grain size was observed in comparison with the pure zinc coating.
\end{abstract}

Keyword: Zeolite Socony Mobil-Five (ZSM-5), Zeolite nano-particles, Corrosion, Composite coating, zinc.

\section{INTRODUCTION}

Nowadays, application of steel in product manufacturing has gained much interest because of its unique properties such as low cost, recyclability and excellent mechanical characteristics. However, low corrosion resistance of this material is the most important problem [1]. One of the most common approaches to overcome this problem is the application of protective coatings to enhance the life span of this material. In this regard various materials have been used as coating such as zinc, cadmium, synthetic and/or extracted organic compounds, modified polymers, resins and alloys [2]. Though cadmium is a good coating material and its corrosion resistance in marine and aerospace conditions is better than steel, but its application is limited due to its toxicity and environmental hazards [2]. Zinc is another metal coating that has a reasonable cost protection and exhibits sufficient air resistance. These properties make zinc a proper coating material but its corrosion in smaller extent is unsuitable. There are many reported procedures in order to avoid the corrosion of zinc coatings such as surface modifications and generation of zinc composites [3]. Though the surface modifications provide better stability and exhibit good mechanical properties but the application of these methods are limited because of their toxicity [4]. Another process is generating zinc composite materials. The aim of research works in this field is to generate new zinc composites that have better corrosion and wear resistance and self-lubricating characteristics [5-9].

*Address correspondence to this author at the Department of Applied Chemistry, Faculty of Science, Shiraz Branch, Islamic Azad University, PO Box 71993-5, Shiraz, Iran; Tel: +98-711-6402715; Fax: +98-711-6412488; E-mail: hrs@iaushiraz.net
Besides these, nanotechnology is of growing importance in many branches of research because of the opportunity for miniaturization and the interesting properties associated with small particle size [10]. In recent decades, nano-structured materials have attracted much attention for their novel electronic, magnetic, optical, chemical, and mechanical properties due to their unique properties which are different from bulkmaterials [11-13].

Considering these facts the co-deposition of the nanoparticles (NPs) is in growing interest because of its increased applicability to make the composite coatings more corrosion resistance, tribological and self-lubricant with improved metallurgical and mechanical properties [14]. Between the wide range of nano-structured materials, carbon nano tubes and nano-sized carbon paste [15], nano-particles of $\mathrm{TiO}_{2}$ and $\mathrm{Fe}_{2} \mathrm{O}_{3}$ $[2,16]$, silica and silicon carbide [17] and ceramic powders are in great demand for the generation of Still composite coatings and have been co-deposited with zinc metal successfully.

Zeolite Socony Mobil-Five (ZSM-5) NPs have some unique characteristics such as unique hape and structure, low dielectric constant (low-k), low cost, facile accessibility, good hardness, high thermal, mechanical and chemical stability makes it an excellent material for reinforcement of metallic coatings. ZSM-5 NPs also improves wear resistance, hardness andother properties such as corrosion resistance. Considering the above facts we now introduce the Zn-ZSM-5 NPs composite as a new, efficient and wear resistance coating material for inhibition of still corrosion.

\section{EXPERIMENTAL SECTION}

\subsection{Apparatus and Analysis}

The chemicals used were of analytical grade. They were purchased from Mreck and Aldrich and used without further 
purification. Deionized water was used for the preparation of solutions. The $\mathrm{pH}$ of the bath solution was adjusted with $10 \%$ hydrochloric acid or sodium carbonate solution. Zinc plate of $99.99 \%$ purity was used as anode. The anode was activated each time by immersing in $10 \% \mathrm{HCl}$ followed by water wash. Mild steel plates (AISI-1079) were mechanically polished to obtain a smooth surface and degreased by trichloroethylene to be used as cathode. The scales and dust on the steel plates were removed by dipping in $10 \% \mathrm{HCl}$ and were subjected to electrocleaning process. These steel plates were washed with water and used for the experiments as such. ZSM-5 NPs zeolite samples were synthesized from kaolin clay as alumina source having a large amount of quartz (39\%) and silicic acid as silica source by hydrothermal treatment with $\mathrm{NaOH}$ in the presence of tetra propyl ammonium hydroxide as a template, according to procedure reported elsewhere [18]. The prepared sample was characterized using XRD and SEM technique. X-ray diffraction (XRD) patterns were measured on a D 500 Siemens X-ray diffractometer using monochromatic $\mathrm{Cu} \mathrm{K} \alpha$ radiation $(\lambda=0.154 \mathrm{~nm})$, operated at $30-40 \mathrm{kV}$ and $40-50 \mathrm{~mA}$. The assignment of the crystalline phases was carried out using PCPDFWIN software (version 2.2) for ICDD database and the relative crystallinity of ZSM-5 was calculated based on the intensity of the peaks of $2 \theta=22-25$. Average crystal size measured by Scherer's equation from XRD peak was between $2 \theta=7-10$. The morphology and crystalline size of the sample was examined under a scanning electron microscope (SEM, LEO 440i) using samples coated with an $\mathrm{Au}$ film. Elemental analysis was carried out using link, ISIS300, Oxford EDS (energy dispersion spectroscopy) detector. The scanning electron micrograph of zeolite having $5 \times 10^{-2}$ (Kaolin/silicic acid \%wt) is shown in Fig. (1) which shows that this zeolite crystallize in spherical shape crystal and $\mathrm{Si} / \mathrm{Al}$ ratio of the synthesized zeolite was determined about 58-60. The particle size of sample is in the range of 20-50 nm and confirmed by scanning electron micrograph (SEM).

Pure zinc and nano-sized zeolite coating were electrolytically deposited on steel from sulphate bath. The constituents of the bath were zinc sulfate $200 \mathrm{~g} / \mathrm{L}$, sodium sulfate $65 \mathrm{~g} / \mathrm{L}$, sodium chloride $40 \mathrm{gL}^{-1}, \mathrm{H}_{3} \mathrm{BO}_{3} 8 \mathrm{gL}^{-1}$, cetyltrimethylammonium bromide $1.75 \mathrm{gL}^{-1}$ and ZSM-5 NPs $6 \mathrm{gL}^{-1}$. The mean diameter of zeolite particles were in the range of 20-40nm. The SEM image and XRD spectra of zeolite are given in Figs. (1 and $\mathbf{2}$ ) respectively.

The bath solution $(267 \mathrm{ml})$ was subjected to magnetic stirring at $200 \mathrm{rpm}$ for $4 \mathrm{~h}$ before plating. After plating experiment, the plates were activated by dipping in $1 \%$ nitric acid for $2 \mathrm{~s}$ followed by water wash. The nature and appearance of zinc and the composite were carefully studied.

The same surface area of anode and cathode was used for electro-deposition process. All the experiments were conducted at $298 \pm 1 \mathrm{~K}$ and $\mathrm{pH} 3.5$. The deposits obtained at a constant current density for plating were $3.5 \mathrm{Adm}^{-2}$. The electro-deposition process under galvanostatic condition using a regulated DC power source was carried out.

The composite coating of Zn-zeolite NPs on steel specimens was electro deposited from different electrolytes containing known amount of constituents.

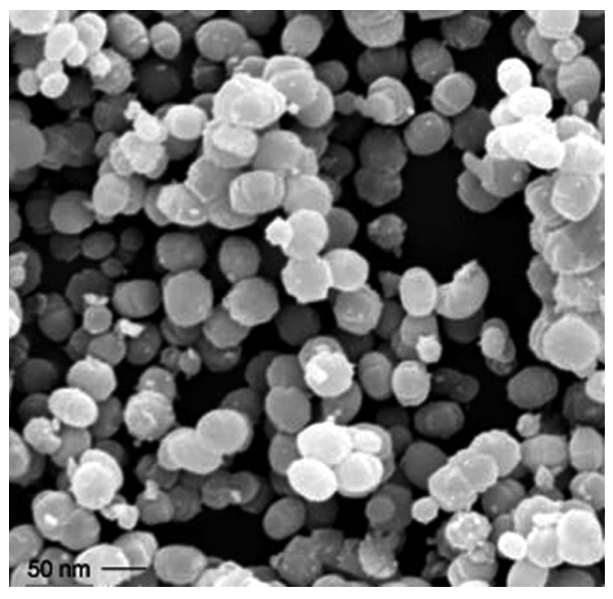

Fig. (1). SEM micrograph of nano-sized ZSM-5 zeolite.

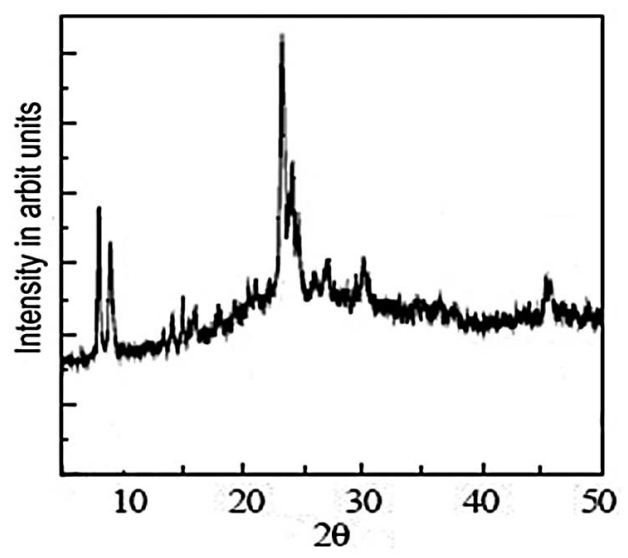

Fig. (2). XRD Spectrum of nano-sized ZSM-5 Zeolite.

The corrosion behavior of zinc coated samples with and without zeolite NPs were studied by weight-loss measurements at $298 \mathrm{~K}$. The exposed area of the specimen was $2 \times 2 \mathrm{~cm}^{2}$. The corrosive medium was $3.5 \% \mathrm{NaCl}$ solution. The coated samples were immersed in $\mathrm{NaCl}$ solution and the plates were weighed periodically for every $24 \mathrm{~h}$ in 15 days. The loss in weight so obtained was used to determine the corrosion rate.

Polarization studies were carried out by using a threecompartment cell. The steel sample coated, with pure zinc or Zn-zeolite NPs was used as working electrode with an exposed area of $1 \mathrm{~cm}^{2}$. Also, platinum wire was used as counter electrode. The cathode potential was recorded galvanostatically with respect to standard calomel electrode as reference electrode at different current densities. The electrolyte was $3.5 \% \mathrm{NaCl}$ solution.

The electrochemical measurements were performed using Electro analyzer from Metrohm made Switzerland. The recorded results were polarization curves at a sweep rate of $0.1 \mathrm{mVs}^{-1}$. The frequency range that the electrochemical impedance recorded was measured from $100 \mathrm{MHz}$ to 10 $\mathrm{mHz}$ with $\pm 5 \mathrm{mV}$ AC amplitude. This was done without any wave generated by a frequency response analyzer. In the same cell with mild steel as cathode cyclic voltammetry studies were carried out. Then the mild steel was 
successfully polished. Once this process was done, then it was cleaned ultrasonically with doubly distilled, deionized water. In this study electrolyte sulphate bath with or without nano zeolite was employed.

An adopting porosity test was performed to estimate the porous natures of the coated specimens. The steel of pure zinc and Zn-zeolite NPs coated samples of $5 \times 5 \mathrm{~cm}^{2}$ area were employed for this work. The coating thickness was in the range of $15-20 \mu \mathrm{m}$. The specimens after plating were subjected to bright dip in 1\% nitric acid, then it was water wash. A Ferroxyl test was done to assess the porosity of the deposit $[19,20]$. This test indicated porous free nature of the deposit. Further, the salt spray test as per ASTM B 117 was carried out in a salt spray chamber [21]. The coated articles were hanged freely in a salt spray chamber, then the test was conducted by continuous spraying of 5\% sodium chloride solution vapors. The corrosion process was facilitated by the drops from the fog of $\mathrm{NaCl}$ that got accumulated on the surface of the articles. It resulted in the formation of zinc salts which is known as white rust. The time required for the formation of white rust was used to indicate the corrosion resistance. The composite coating delays the formation of white rust due to the high corrosion resistance property. The final result is an increase in the durability of articles. The specimens' surfaces were observed carefully and the duration of time for the formation of white rust was noted.

The Vickers micro-hardness of the deposit was determined by an indentation technique with a weight of $50 \mathrm{~g}$ for 10s using Newage micro-hardness NI-HV30 testing systems, made in USA. This test replicated five times and the average values were recorded. The morphology study of the surface of coated samples, before and after corrosion tests, was done by scanning electron microscopy (SEM).

\section{RESULTS AND DISCUSSIONS}

\subsection{Gravity Loss Measurements}

The weight loss profiles of sole zinc and Zn-ZSM-5 NPs coated samples which were due to forced corrosion process in $\mathrm{NaCl}$ medium were studied and the obtained results are summarized in Fig. (3). The weight loss in Zn-ZSM-5 NPs coated sample were about half lower than pure zinc coated sample. This observation shows a good reinforcing action of ZSM-5 NPs that was easily merged in zinc matrix [22]. ZSM-5 NPs postponed the easy dissolution of the zinc and consequently the corrosion rate was reduced in composite coating. As can be seen from the Fig. (3), the presence of ZSM-5 NPs in the coating decreased the dissolution of zinc and hence decreased the corrosion rate. In both cases the corrosion rate differences were almost the same throughout the 360 hour.

A plausible mechanism for the weight loss reaction in the presence of ZSM-5 NPs is depicted in Schemes $\mathbf{1}$ and 2. Since the crystallization of ZSM-5 is associated with near concomitant appearance and a sudden increase in electrical conductivity [23] the majority of the conductivity measured should be attributed to the free and associated small ions such as $\mathrm{Na}^{+}$and $\mathrm{OH}^{-}$. During crystallization, condensation reactions occur and release the bonded hydroxyl groups along with the associated cations as free $\mathrm{Na}^{+}$and $\mathrm{OH}^{-}$or $\mathrm{TPA}^{+}$and $\mathrm{OH}^{-}$, resulting in an increase in electrical conductivity. The reverse of condensation reaction (depolymerization) as $\mathrm{NaOH}$ or $\mathrm{NaCl}$ attack destroy the colloidal silica(zeolite structure)and accelerate the rate of corrosion depending on the crystal structure of zeolite and the amount of silica content in zeolite constituents as shown in Scheme $\mathbf{1}$ for low-silica zeolite systems in which Si-O-Al bonds are dissociated. The bond cleavage reaction in which $\mathrm{Si}-\mathrm{O}-\mathrm{Si}$ bonds are dissociated, as in high-silica zeolite systems such as ZSM-5 occurs is shown in Scheme 2.

The often-practiced process of gel aging has been monitored with electrical conductivity measurement. The electrical conductivity of the ZSM-5 synthetic gel during aging at room temperature is shown in ref. [23]. The electrical conductivity declines monotonically, reaching an asymptote after several hours. The decrease in electrical conductivity confirmed the above theory.

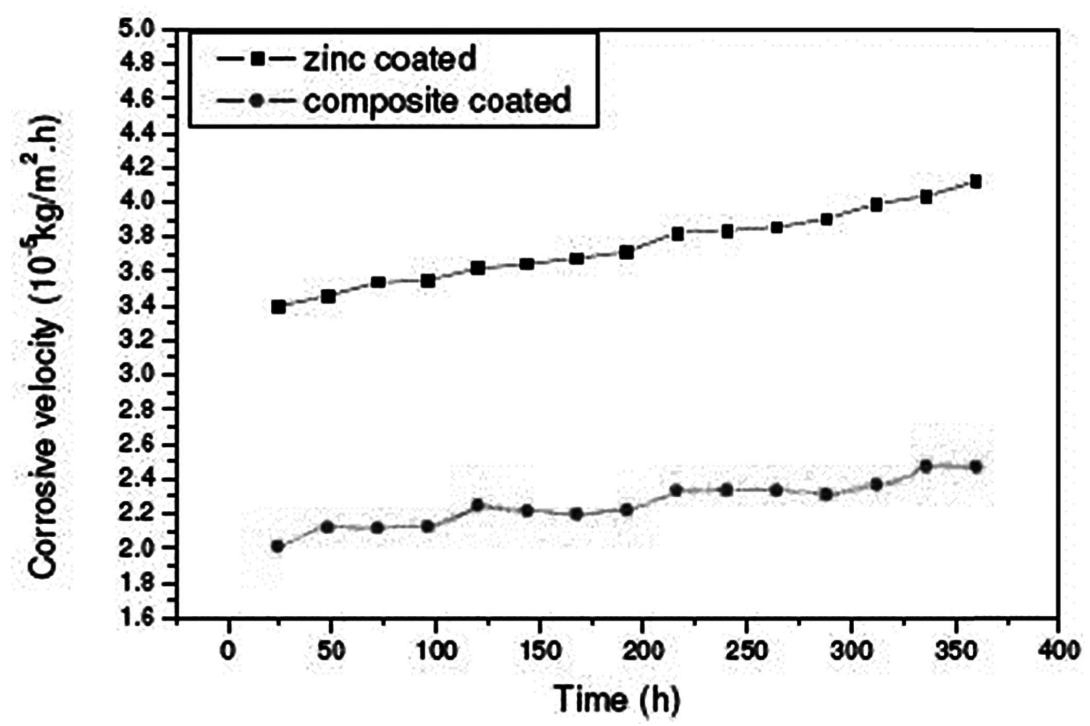

Fig. (3). Corrosion rate with immersion time for pure zinc coating and Zn-ZSM-5 NPs composite coating samples in $3.5 \%$ sodium chloride solution. 


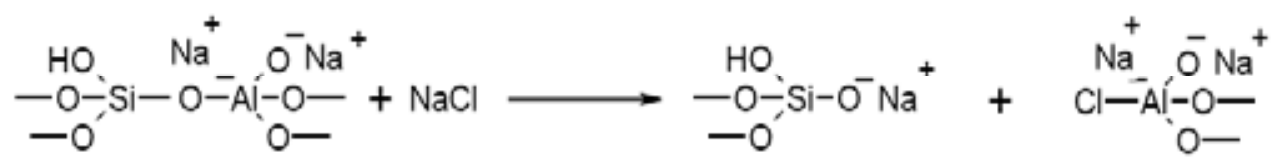

Scheme 1. Low-silica zeolite systems in which Si-O-Al bonds are dissociated.

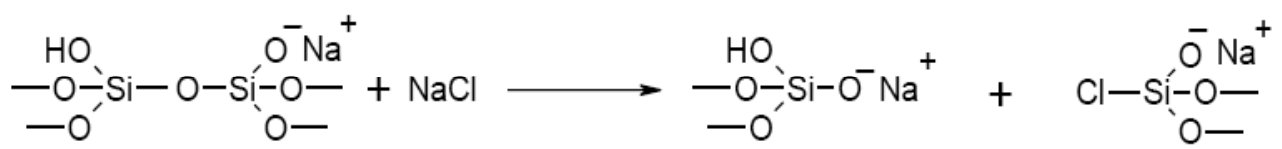

Scheme 2. High-silica zeolite systems in which Si-O-Si bonds are dissociated.

Finally after several days, when the corrosion process led to a perforation of the zinc layer and the cathodic protection mechanism of the zinc for the steel substrate started, $\mathrm{Zn}$ as $\mathrm{Zn}{ }^{2+}$ left the substrate and weight loss began.

\subsection{Test of Salt Spraying}

The industrial method of appraising the corrosion treatment for prediction of the durability of zinc-plated parts was salt spraying test. The test was conducted by spraying $5 \% \mathrm{NaCl}$ solution vapors on coated articles hanged freely in a closed chamber. The drops from the fog of $\mathrm{NaCl}$ get accumulated on the surface of the particles and facilitate the corrosion resulting in the formation of zinc salts called white rust. The number of hours required for the formation of white rust was the indicator of the corrosion resistance. The higher corrosion resistance of the coating delays the production of white rust. In this study the white rust was seen after 22 hours on pure zinc coating and the Zn-ZSM-5 NPs composite indicated the white rust after 48 hours. This test also confirms the enhancement of corrosion resistance property of zinc in the presence of nano-sized zeolite. An interesting question is what underlying chemical reactions has taken place on the surface of sample and what are the factors causing corrosion on the surface. Hashimoto et al., [24] studied the microstructure of electrodeposited $\mathrm{Zn}-\mathrm{SiO}_{2}$ composites before and after corrosion tests. They described after a wet and dry cyclic corrosion test, including $\mathrm{NaCl}$ solution spray, zinc hydroxide, zinc oxide and zinc hydroxylchloride as white corrosion products were formed on the surface of the $\mathrm{Zn}-\mathrm{SiO}_{2}$ composites. The high corrosion resistance of the composites is attributed to the formation of protective corrosion products in the composite layer supported by $\mathrm{SiO}_{2}$. They showed the diffraction intensity of the corrosion products, detected in the zinc deposit, is higher than the $\mathrm{Zn}-\mathrm{SiO}_{2}$ composite. Therefore, the result implies that the corrosion rate of the $\mathrm{Zn}-\mathrm{SiO}_{2}$ composite is lower than that of the zinc deposit.

Azizi et al., [25] co-deposited different particles of silica flakes and mica (phyllosilicate minerals) with zinc on the steel. They tested the corrosion resistance composite layers and compared the results with sole zinc coating. Therefore, the pure zinc with three different concentrations of silica was compared. The test was made by keeping the samples in a $4 \% \mathrm{NaCl}$ solution at $25^{\circ} \mathrm{C}$ and by measuring the free corrosion potential as a function of time. At the beginning the measured potentials were similar to the free corrosion potential of the pure zinc. After a while, white corrosion products were observed for all samples. After several days, when the corrosion process led to a perforation of the zinc layer and the cathodic protection mechanism of the zinc for the steel substrate started, a shift of the free corrosion potential towards the free corrosion potential of the steel was observed. The shift began in the composite layers. The layers with the highest particle concentration showed the slowest shift. They believed that the disc shaped particles act as diffusion barrier for $\mathrm{H}_{2} \mathrm{O}$ and $\mathrm{O}_{2}$ and a similar barrier protection mechanism acts in the zinc composite coatings in connection with the cathodic protection mechanism by the zinc matrix. There have been similar reports about aluminumoxide [25]. Since ZSM-5 also is an aluminosilicate, therefore the high corrosion resistance of the Zn-ZSM-5 composite coating as mentioned above is attributed to the chemical and physical properties of composite. Due to the usage of nano-sized zeolite the composite coating was compacted and acted as diffusion barrier for $\mathrm{H}_{2} \mathrm{O}$ and $\mathrm{O}_{2}$. On chemical aspect the corrosion resistance of the $\mathrm{Zn}$ film including zeolite nanoparticles increased because zeolites as charged exchange have low electrical conductivity in comparison with $\mathrm{Zn}$ films. Specific crystal structure of ZSM-5 [26] also has influenced the corrosion resistivity of nano-composite film.

\subsection{Electrochemical Scales}

The potential was measured under galvanostatic conditions, at different current densities with respect to saturated calomel electrode using the bath solution with and without ZSM-5 NPs. Anodic polarization curves show that the potential of composite coated sample was more positive in the entire current density region when compared to pure zinc coated sample except in the beginning. The anodic polarization curves of pure zinc and composite coated samples are shown in Fig. (4). The open circuit potential for Zn-ZSM-5 coated sample (-1.035 V) was almost the same as that of pure zinc coated sample $(-1.032 \mathrm{~V})$. In zinc coated sample the current density values increase sharply at the potential region -0.99 V. For composite coated sample there was no sharp increment in current density values but it was slow. It shows that metal dissolution happens continually at lower potential values in zinc coated sample while in composite coated sample dissolution of metal takes place at higher anode potential. Moreover, there was continuous rise in current density resulting in the steady state increase in 


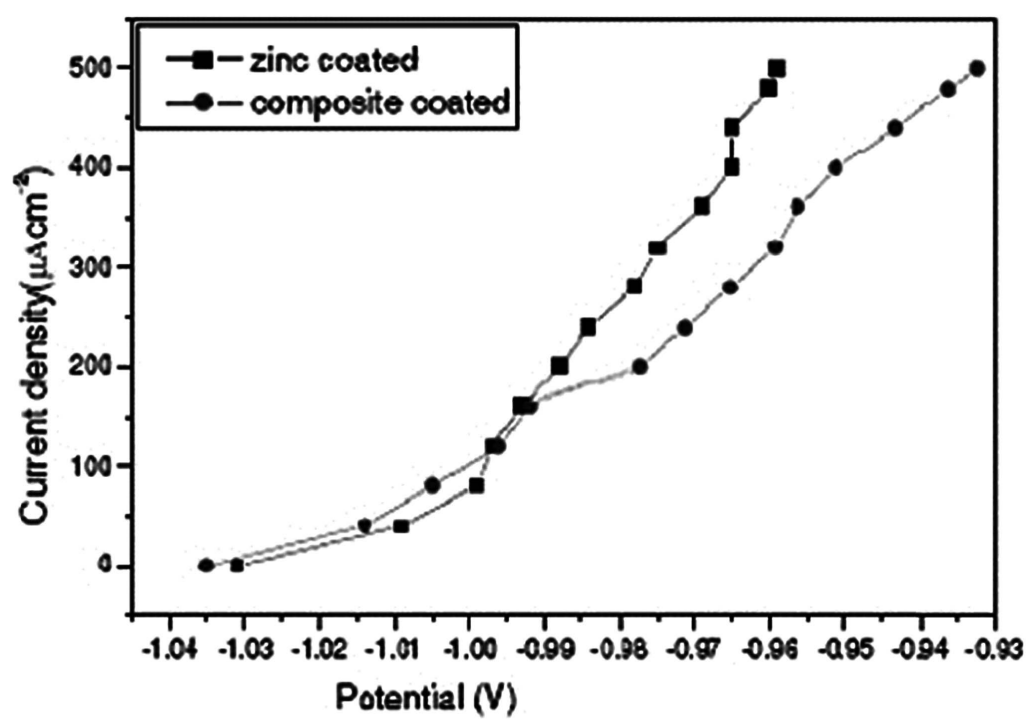

Fig. (4). Anodic polarization diagrams for pure zinc coating and ZSM-5 composite coating sample in 3.5\% sodium chloride solution.

dissolution rate of metal. It could be concluded that intermixing of ZSM-5 NPs provides higher resistance for dissolution of zinc.

Impedance spectra were recorded for pure zinc coated and composite coated and is shown in Fig. (5). It shows that Nyquist plot was observed for the pure zinc and composite samples. Zn-ZSM-5 NPs composite coated samples indicated higher $R_{p}\left(8.5 \Omega \mathrm{cm}^{2}\right.$ against $\left.7.1 \Omega \mathrm{cm}^{2}\right)$ values and hence the ZSM-5 incorporation offered higher polarization resistance. The $\mathrm{C}_{\mathrm{dl}}$ values, $3.12 \times 10^{-5} \mu \mathrm{F} \mathrm{cm}^{-2}$ for composite against $4.96 \times 10^{-5} \mu \mathrm{F} \mathrm{cm}$ for pure zinc, were also found in accordance with these observations.

The cyclic voltagrams of pure electrolyte and electrolyte containing ZSM-5 NPs are shown in Fig. (6). The presence of ZSM-5 NPs indicates the lesser cathodic current and provides obvious reason that it get adsorbed onto the active sites of the coating. Also, a small amount of zinc deposited at lower potentials raises the overpotential for hydrogen evolution, resulting in lower cathodic current. Also, in anodic region current is reduced. This shows that the zinc dissolution was somehow difficult in the presence of ZSM-5 NPs in its coating.

\subsection{Micro-Hardness Scales}

The micro-hardness values were also studied. Based on Hall-Petch Law there is inverse relationship between the amounts of micro-hardness properties with particle size. In this case also due to the presence of ZSM-5 NPs the grain size of the coating was decreased, therefore micro-hardness values increased. A micro-hardness value of composite coated sample was 112 against 72 of zinc coating. In addition to the above, existence of higher percentage of $\mathrm{Si}$ atom or silicone oxide in ZSM-5 structure causes the increase in microhardness of composite coating over pure zinc.

\subsection{Superficies Morphology}

Fig. (7) shows the XRD patterns of the electrodeposited pure zinc and Zn-ZSM-5 NPs composite coating. The average size of crystals of the Zn-ZSM-5 NPs composite

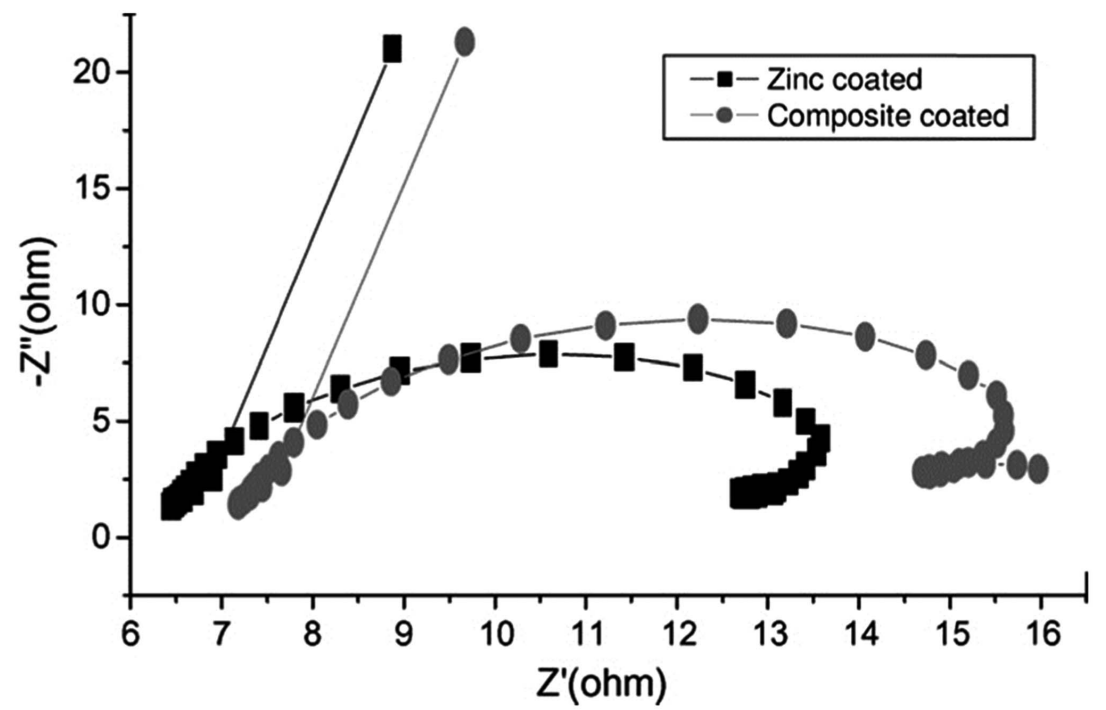

Fig. (5). Impedance diagrams for pure zinc coating and Zn-ZSM-5 coating samples in $3.5 \%$ sodium chloride solution. 


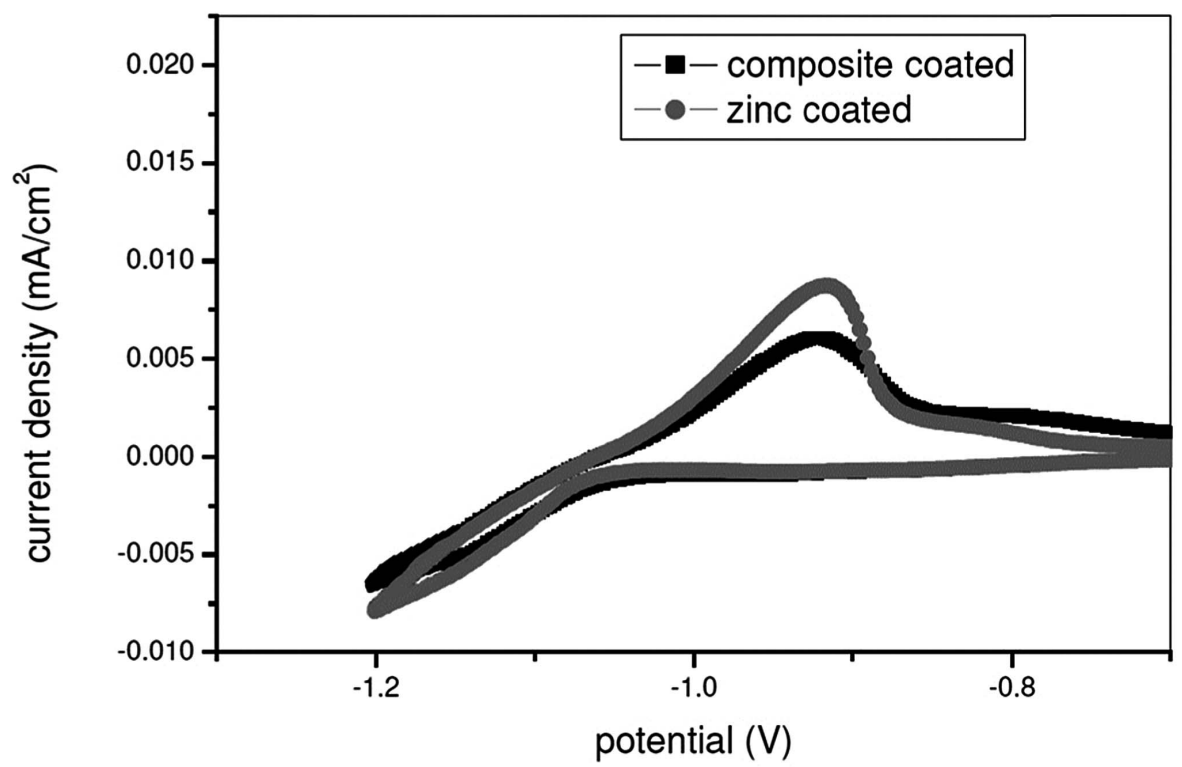

Fig. (6). Cyclic voltagrams of bath solution for pure zinc coating and Zn-ZSM-5 coating samples.
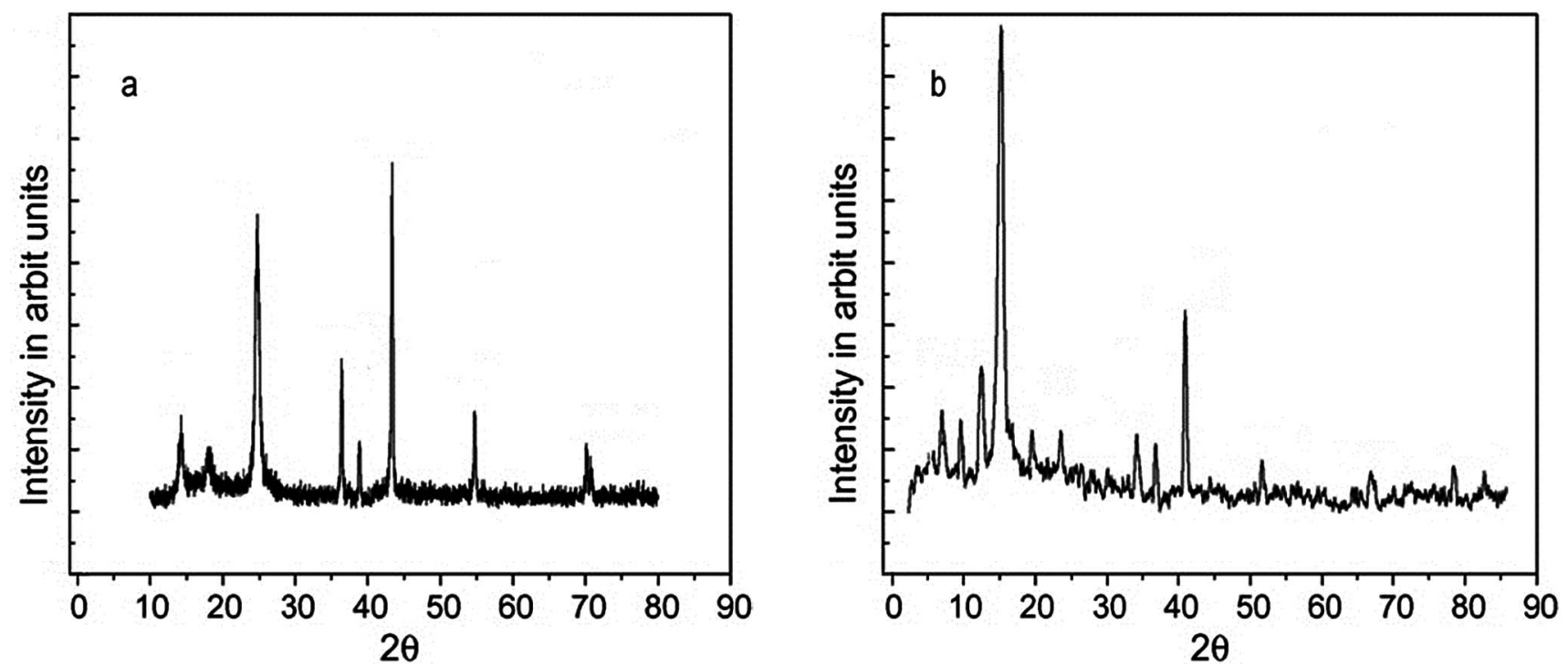

Fig. (7). XRD of pure zinc coating (a) and Zn-ZSM-5 coating sample (b).
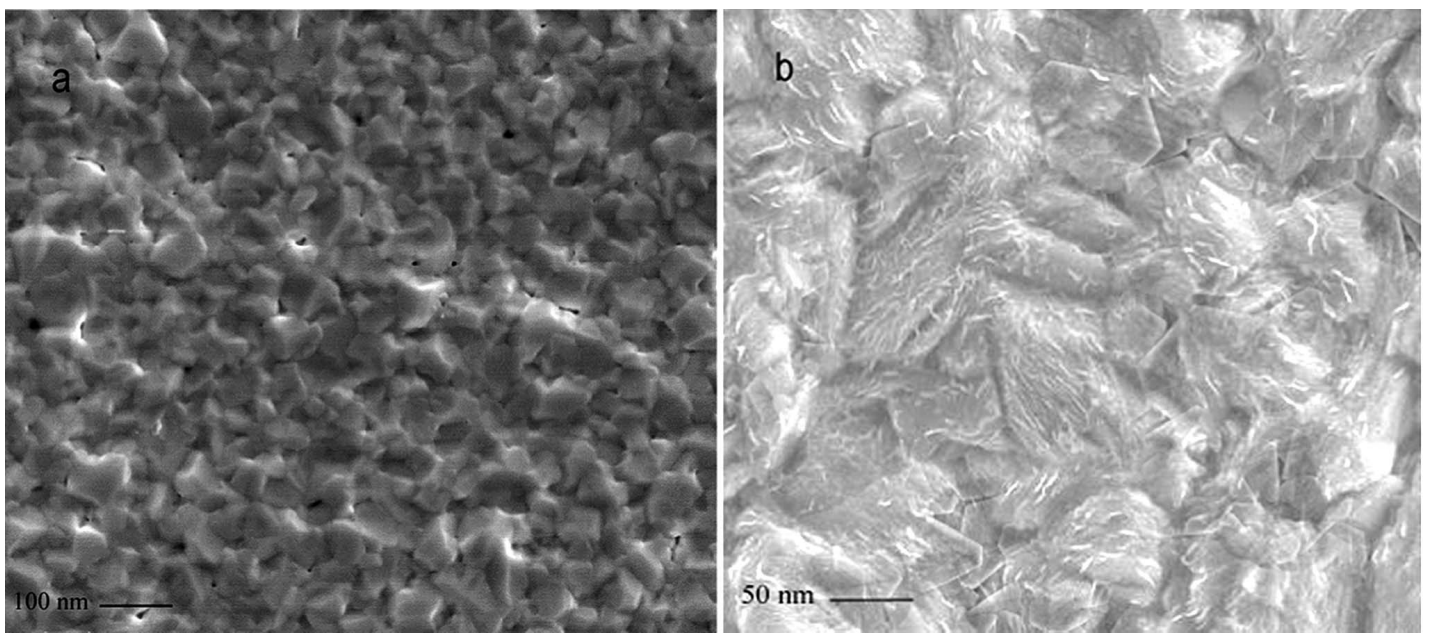

Fig. (8). SEM images of pure zinc coating (a) and Zn-ZSM-5 coating sample (b). 

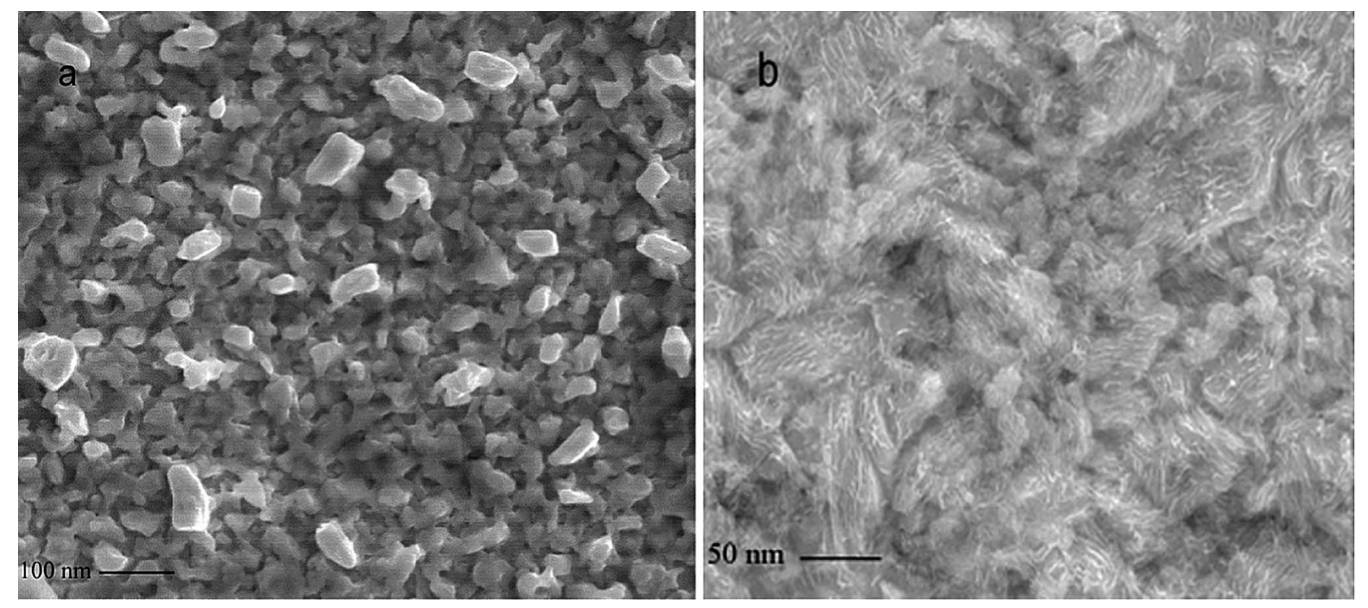

Fig. (9). SEM images of pure zinc coating (a) Zn-ZSM-5 coating (b) after fifteen days in corrosion experiment.

coating was $27 \mathrm{~nm}$ against $36 \mathrm{~nm}$ for pure zinc coating. This is due to the decrease in the grain size of the Zn-ZSM-5 NPs composite deposit. This was also seen in SEM images. The SEM images of pure zinc coated and Zn-ZSM-5 NPs coated samples are shown in Fig. (8). The grain size of the pure zinc coated sample was larger when compared with the composite coating. The presence of ZSM-5 provides more nucleation sites and slows the crystal growth and therefore composite coated surface possess smaller grain size. During electrodeposition the crystal size was monitored by the rate of fresh nuclei formation or rate of growth of crystal. Fine grained deposit was obtained at higher rate of formation of nuclei. The addition of zeolite particles might provide a large number of cathodic sites and eventually more number of fresh nuclei formed on metal surface.

Fig. (9a and $\mathbf{b}$ ) shows the SEM images of pure zinc coated and composite coated samples after corrosion experiments. The coated specimens were immersed in $3.5 \%$ $\mathrm{NaCl}$ for $360 \mathrm{~h}$. The SEM image of zinc coated sample showed the corrosion products (white rust) on the whole surface, but composite coated sample exhibited lesser amount of corrosion products. The metal surface possesses defects, cracks, gaps, crevices and micro-holes which were generally larger than micron. It was obvious that the NPs easily enter and fill these defects. In the present case also the zeolite NPs enters and fills these gaps of the surface of zinc. Moreover, this micro-hole behaves as active sites for dissolution of metal during corrosion. Thus, these holes were covered in Zn-ZSM-5 NPs composites by ZSM-5 NPs thereby bringing down the corrosion rate. Therefore, as it can be seen in Fig. (9a and $\mathbf{b}$ ) in the presence of ZSM-5 NPs, the localized corrosion was inhibited and mainly homogenous corrosion occurred. These results indicated that the composite coated sample did not offer any retroversion even after 15 days of immersion in $\mathrm{NaCl}$ and retained higher corrosion resistance property.

\section{CONCLUSIONS}

Electrochemical studies indicated that Zn-ZSM-5 NPs composite coating exhibited much lower activity than pure zinc coating. Therefore, they retain better stability in the external environment. The incorporation of ZSM-5 NPs in the coating led to improvement in the crystal size and enhances corrosion resistance and micro-hardness of the composite coating as compared to the pure zinc coatings. By contrast to the conventional $\mathrm{Zn}$ and $\mathrm{Zn} / \mathrm{Alloy}$ coating this fine-grained nano-scaled zeolite with rich firm constituents like Si atom improve coating and show high performance chemical and electrochemical corrosion resistance properties. The SEM images indicated ZSM-5 NPs uniformly distributed in the zinc coating and also in Zn-ZSM-5 NPs composite coating corrosion rate was smaller than conventional coating. This excellent corrosion resistance and micro-hardness of Zn-ZSM-5 NPs composite coating provides wide applications in modern industry.

\section{ACKNOWLEDGEMENT}

The authors express their deepest sense of appreciation to professor Jong-Sung Yu for his inspiring suggestions and helpful advice and thank the research council of Islamic Azad University, Shiraz branch for financial support of this work.

\section{REFERENCES}

[1] Prabhu, R. A.; Shanbhag, A. V.; Venkatesha, T. V. Influence of tramadol [2- [(dimethylamino)methyl]-1-(3-methoxyphenyl) cyclohexanol hydrate] on corrosion inhibition of mild steel in acidic media. J. Appl. Electrochem., 2007, 37, 491-497.

[2] B. M. Paraveen and T. V. Venkatesha, Electrodeposition and properties of $\mathrm{Zn}$-nanosized $\mathrm{TiO}_{2}$ composite coatings. Appl. Surface Sci., 2008, 254, 2418-2424.

[3] Zhang, X. G. Corrosion and Electrochemistry of Zinc, New York: Plenum Publishing Corporation, 1996.

[4] Praveen, B. M.; Venkatesha, T. V.; Arthoba Naik, Y.; Prashantha, $\mathrm{K}$. Corrosion studies of carbon nanotubes-Zn composite coating. Surf. Coat. Technol., 2007, 201, 5836-5842.

[5] Roos, J. R.; Celis, J. P.; Fransaer, J.; Buelens, C.; The development of composite plating for advanced materials: overview. JOM, 1990, 42, 60-63.

[6] Celis, J. P.; Roos, J. R.; Buelens, C.; Fransaer, J. Mechanism of electrolytic composite plating: Survey and trends. Trans. Inst. Met. Finish., 1991, 69, 133-139.

[7] Buelens, C.; Fransaer, J.; Celis, J. P.; Roos, J. R. The mechanism of electrolytic codeposition of particles with metals: a review. Bull. Electrochem., 1993, 8, 371-375.

[8] Fransaer, J.; Celis, J.P.; Roos, J. R. Mechanisms of composite electroplating. Trans. Institute Metal Finish., 1993, 91, 97-102.

[9] Hovestad, A.; Jansen, L. J. J. Electrochemical codeposition of inert particles in a metallic matrix. J. Appl. Electrochem., 1995, 25, $519-523$. 
[10] Rautio, J.; Perämäki, P.; Honkamo, J.; Jantunen, H. Effect of synthesis method variables on particle size in the preparation of homogeneous doped nano ZnO material. Microchem J., 2009, 91, 272-276.

[11] Xia, Y. N.; Yang, P. D.; Sun, Y. G.; Wu, Y. Y.; Mayers, B.; Gates, B.; Yin, Y. D.; Kin, F.; Yan, H. Q. One-dimensional nanostructures: synthesis, characterization, and applications. Adv. Mater., 2003, 15, 353-389.

[12] Wang, Z. L.; Kong, X. Y.; Ding, Y.; Gao, P. X.; Hughes, W. L.; Yang, R.; Zhang, Y. Semiconducting and piezoelectric oxide nanostructures induced by polar surfaces. Adv. Funct. Mater., 2004, 14, 943-956.

[13] Chae, W.S.; Shin, H.W.; Lee, E.S.; Shin, E. J.; Jung, J. S.; Kim, Y. R. Excitation dynamics in anisotropic nanostructures of star-shaped CdS. J. Phys. Chem. B, 2005, 109, 6204-6209.

[14] Musiani, M. Electrodeposition of composites: an expanding subject in electrochemical materials science. Electrochim. Acta, 2000, 45, 3397-3402.

[15] Paraveen, B. M.; Venkatesha, T. V. Generation and corrosion behavior of Zn-nano sized carbon black composite coating. Int. J. Electrochem. Sci. 2009, 4, 258-266.

[16] Shibli, S. M. A.; Dilimon, V. S.; Antony, S. P.; Manu, R. Incorporation of $\mathrm{TiO}_{2}$ in hot dip zinc coating for efficient resistance to biogrowth. Surf. Coat. Technol., 2006, 200, 4791-4796.

[17] Shi, L.; Sun, C.; Gao, P.; Zhou, F.; Liu, W. Mechanical properties and wear and corrosion resistance of electrodeposited $\mathrm{Ni}-\mathrm{Co} / \mathrm{SiC}$ nanocomposite coating. Appl. Surf. Sci. 2006, 252, 3591-3599.
[18] Khatamian, M.; Irani, M. Preparation and characterization of nanosized ZSM-5 zeolite using kaolin and investigation of kaolin content, crystallization time and temperature changes on the size and crystallinity of products. J. Iran. Chem. Soc., 2009, 6(1), 187-194.

[19] Sachin, H. P.; Achary, G.; Naik, A.; Venkatesha, T. V. Polynitroaniline as brightener for zinc-nickel alloy plating from non-cyanide sulphate bath. Bull. Mater. Sci., 2007, 30, 57-64.

[20] Available from: http://www.lmats.com.au/services/chemical-testing/ contamination-testing/ferroxyl-test.html

[21] Arthoba, N.; Venkatesha, T. V. A new condensation product for zinc plating from non-cyanide alkaline bath. Bull. Mater. Sci., 2005, 28, 495-502.

[22] Chen, X. H.; Chen, C.S.; Xiao, H.N.; Cheng, F.Q.; Zhang, G.; Yi, G. J. Corrosion behavior of carbon nanotubes-Ni composite coating. Surf. Coat. Technol., 2005, 191, 351-356.

[23] Cao, G.; Shah, M. J. In situ monitoring of zeolite crystallization by electrical conductivity measurement: New insight into zeolite crystallization mechanism. Microporos. Mesoporos. Mater., 2007, 101, 19-23.

[24] Hashimoto, S.; Abe, M.; The characterization of electrodeposited $\mathrm{Zn}-\mathrm{SiO}_{2}$ composites before and after corrosion test. Corros. Sci, 1994, 36, 2125-2137.

[25] Azizi, M.; Schneider, W.; Plieth, W.; Electrolytic co-deposition of silicate and mica particles with zinc, J. Solid State Electrochem., 2005, 9, 429-437.

[26] Kulprathipanja, S. Zeolites in Industrial Separation and Catalyst, WILEY-VCH Verlag GmbH \& Co. KGaA, Weinheim 2010.

This is an open access article licensed under the terms of the Creative Commons Attribution Non-Commercial License (http://creativecommons.org/licenses/ by-nc/3.0/) which permits unrestricted, non-commercial use, distribution and reproduction in any medium, provided the work is properly cited. 